\title{
Behavioral Analysis of Internal Memory States Using Cooling-induced Retrograde Amnesia in Limax flavus
}

\author{
Atsushi Yamada, Tatsuhiko Sekiguchi, Haruhiko Suzuki, and Atsuo Mizukami \\ Sanyo Electric Company, Limited, Tsukuba Research Center, Tsukuba, Ibaraki 305, Japan
}

\begin{abstract}
Temporal evolution of internal memory states in a terrestrial mollusk, Limax flavus, was studied using cooling-induced retrograde amnesia. The slug was conditioned to avoid carrot odor by temporally correlated presentation of carrot juice and a bitter-taste stimulus of quinidine sulfate. We could induce retrograde amnesia by cooling of the conditioned slug immediately after the training trial. Thus, we studied the memory states in the slug using the retrograde amnesia according to strategies used in the studies of memory states in mammals or insects.
\end{abstract}

In the early process of memory acquisition, at least two distinctive memory states were observed, short-term memory and long-term memory (LTM). For LTM, two states were also observed. One was a reactivated state of $L T M$, which was sensitive to the cooling used to induce the amnesia. The other was a so-called resting state of LTM, which was insensitive to cooling. A few days after memory acquisition, further evolution was observed in that the amnesia could not be induced even if the memory trace was reactivated. The results obtained in Limax flavus was comparable with those obtained in a variety of animals.

Because of their relative simplicity, molluscan nervous systems have provided an advantageous model system for studies of the cellular mechanisms of nonassociative or associative learning (Carew and Sahley, 1986; Byrne, 1987). One important and interesting question is whether the principles emerging from studies of molluscan nervous systems can be applied to other animals, especially mammals. Gclpcrin and his colleagues partly answered this question by demonstrating that a terrestrial slug, Limax, showed some logical operations similar to those known in vertebrates, such as first- and second-order conditioning, blocking (Sahley et al., 1981b; Gelperin et al., 1985), one-trial associative learning (Sahley et al., 1981a), and appetitive learning (Sahley et al., 1990). They also showed a similarity between the slug and vertebrates in associative structure obtained by second-order conditioning (Sahley et al., 1984). These results suggest a degree of parallelism between mollusks and vertebrates in the mechanisms of learning and memory.

Another approach for examining the above question is to

\footnotetext{
Received Mar. 12, 1991; revised Oct. 8, 1991; accepted Oct. 10, 1991.

We gratefully acknowledge the helpful advice of Dr. Masafumi Yano, Tokyo University.

Correspondence should be addressed to Tatsuhiko Sekiguchi, Sanyo Electric Co., Ltd., Tsukuba Research Center, 2-1 Koyadai, Tsukuba, Ibaraki 305, Japan. Copyright $\odot 1992$ Society for Neuroscience $0270-6474 / 92 / 120729-07 \$ 05.00 / 0$
}

search for similarities in the temporal evolution of the memory state. It is widely accepted that in a variety of animals memory changes its state in a time-dependent manner (Menzel, 1984; Squire, 1987). In mollusks, the transition from short-term memory (STM) to long-term memory (LTM) has been reported in several species such as Aplysia and Hermissenda (e.g., Pinsker et al., 1973; Crow and Alkon, 1978; Crow, 1983; Frost et al., 1985), where the transition is dependent on the number of training trials or on the training schedules employed. These memory states were also distinguishable using an inhibitor of protein synthesis (Montarolo et al., 1986) or by counting the number of varicosities of identified neurons (Baily and Chen, 1988). However, these expcrimental stratcgics werc considerably different from those employed with mammals or insects as described below. Thus, it has not been possible to compare the results obtained in mollusks with those obtained in animals.

In mammals or insects, experiments on the temporal evolution of the memory state have been carried out mainly using two strategies. One involves observing memory retention from the very early phase of learning. In the case of color-discriminative learning in the honeybee, Apis mellifera carnica, there is a biphasic retention curve consisting of two peaks, which are attributable to STM and LTM (Menzel, 1984). The other strategy is to observe the interference of memory acquisition with a certain stimulus after training trials, that is, induction of retrograde amnesia. The application of electroconvulsive shock (ECS) (e.g., Heriot and Coleman, 1962; McGaugh, 1966), carbon dioxide gas (Taber and Banuazizi, 1966; Freckleton and Wahlstan, 1968), or cooling (Erber, 1976; Quinn and Dudai, 1976) to an animal immediately after a single training trial has been shown to suppress the retention of the learned task. However, such amnesia is not induced when these procedures are applied several minutes or several tens of minutes after the training. Thus, one can distinguish memory states in the early process of memory acquisition using retrograde amnesia, one state (STM) being sensitive and the other (LTM) not. Several workers have reported further evolution of memory states using this type of amnesia (Misanin et al., 1968; Squire and Spanis, 1984). Thus, in mammals or insects, retrograde amnesia has provided a powerful tool for studying the temporal evolution of the memory state. In mollusks, however, there have been no observations of retrograde amnesia or studies on the memory state using amnesia.

In the present study, retrograde amnesia was induced by cooling in Limax flavus after conditioning to avoid food odor. Using the amnesia, we analyzed the retention of memory in the animal. The results that show changes in memory state with time are comparable with those obtained in a variety of animals. 


\section{Materials and Methods}

Subjects. Specimens of Limax flavus were maintained in laboratory culture on frog chow (Oriental Yeast Co. Ltd.) with a light-dark cycle of $14 \mathrm{hr} / 10 \mathrm{hr}$ at $19^{\circ} \mathrm{C}$. Prior to the start of experiments, about 40 slugs were housed in a plastic container $(350 \times 255 \times 62 \mathrm{~mm})$ lined with river sand and were allowed continuous access to the diet. One week before the start of training, animals weighing $1.5-2.0 \mathrm{gm}$ were placed individually into other plastic containers $(113 \times 105 \times 28 \mathrm{~mm})$ lined with moistened filter paper and then starved until the start of the experiments.

Materials used for stimulation. Carrot juice was made in the laboratory. Several carrots were ground with a blender and centrifuged for $30 \mathrm{~min}$ at $7000 \times \mathrm{g}$. The supernatant was used as carrot juice, which was kept at $-20^{\circ} \mathrm{C}$ until use. In the training trial, the carrot juice was applied to moistened filter paper and used as a conditioned taste stimulus (CS). A saturated solution of quinidine sulfate $(1 \mathrm{gm} / 90 \mathrm{ml}$ saline) was applied to moistened filter paper and used as a bitter-taste unconditioned stimulus (US). The saline had the following composition (in mM): $\mathrm{NaCl}, 52.9 ; \mathrm{KCl}, 4.0 ; \mathrm{CaCl}_{2}, 7.0 ; \mathrm{MgCl}_{2}, 4.6 ; \mathrm{KH}_{2} \mathrm{PO}_{4}, 0.2 ; \mathrm{NaHCO}_{3}$, 2.5 ; dextrose, $5 ; \mathrm{pH} 7.6$. The quinidine sulfate was obtained from Wako Pure Chemical Industries, Ltd., and the other reagents were of analytical grade.

First-order conditioning. The procedure for first-order conditioning (FOC) was basically the same as that used in Limax maximus (Sahley et al., 1981a). First, slugs were transferred with tweezers to a plastic container lined with filter paper moistened with carrot juice (C). After a 2 min exposure to the carrot juice, the slugs were directly transferred with tweezers to another plastic container lined with filter paper thoroughly moistened with quinidine sulfate $(Q)$. They were held in contact with the quinidine sulfate for $1 \mathrm{~min}$, then rinsed with 'saline for $5 \mathrm{sec}$ and returned to their individual containers. This paired presentation of CS and US was repeated one to four times with a $2 \mathrm{hr}$ intertrial interval (ITI). Slugs in the control group received the same number of CS and US presentations as those in the FOC group, but the CS-US interstimulus interval (ISI) was $30 \mathrm{~min}$. These FOC procedures are abbreviated hereafter as (CQ) $k$ ( $k$, number of training pairs).

As is described below, the conditioning was assessed in terms of changes in carrot "odor" preference. However, the conditioning was carried out with carrot juice as CS, where the slug was able to sense both "odor" and "taste." Thus, it was important to examine the contribution of carrot taste to the conditioning. At the start of the training trial, 444 of the 468 slugs (94.9\%) ingested the carrot juice. After two CQ training pairs, where the slug showed fairly good performance in the odor-aversive conditioning, 409 of the 468 slugs (84.4\%) still ate the carrot juice. This result suggested that Limax flavus showed odortaste conditioning much more readily than taste-taste conditioning, and that the contribution of taste-taste conditioning was small in the present study. In fact, slugs conditioned with carrot odor as CS showed a similar degree of conditioning to those conditioned with the odor plus taste as CS (data not shown). Sahley et al. (1981a,b) also carried out two types of conditioning and showed that Limax maximus learned to avoid carrot odor by both conditioning methods. Thus, we concluded that taste-taste conditioning did not contribute significantly to the degree of conditioning.

Cooling of the animals. In the experiments involving cooling-induced retrograde amnesia, slugs were cooled to about $1^{\circ} \mathrm{C}$. Each animal was kept in an individual container and left for $5 \mathrm{~min}$ in the freezer compartment of a refrigerator. Within $3 \mathrm{~min}$ in the freezer compartment, the animal ceased its motor activity and the body temperature became about $1^{\circ} \mathrm{C}$ as determined by a thermocouple. This cooling procedure is termed "F."

Testing and the measure of response. The test apparatus (Fig. 1) consisted of three chambers, two of which were for carrot juice and frog chow as odor sources. The carrot odor was generated with a filter paper moistened with carrot juice, and the odor of frog chow was generated with gel containing the powdered chow. These odor sources were placed on the floor of each side chamber. The slug was placed in the center chamber, the walls of which were perforated. Thus, the animal could sense the food odors but could not eat their sources. A line divided the center chamber into a "carrot side" and a "chow side."

In each testing trial, a slug was placed in the center chamber with its body aligned along the center line. When the slug's head crossed the center line, the time it spent on the carrot side was recorded for the next 120 sec (a small point on the head between the tentacles, shown

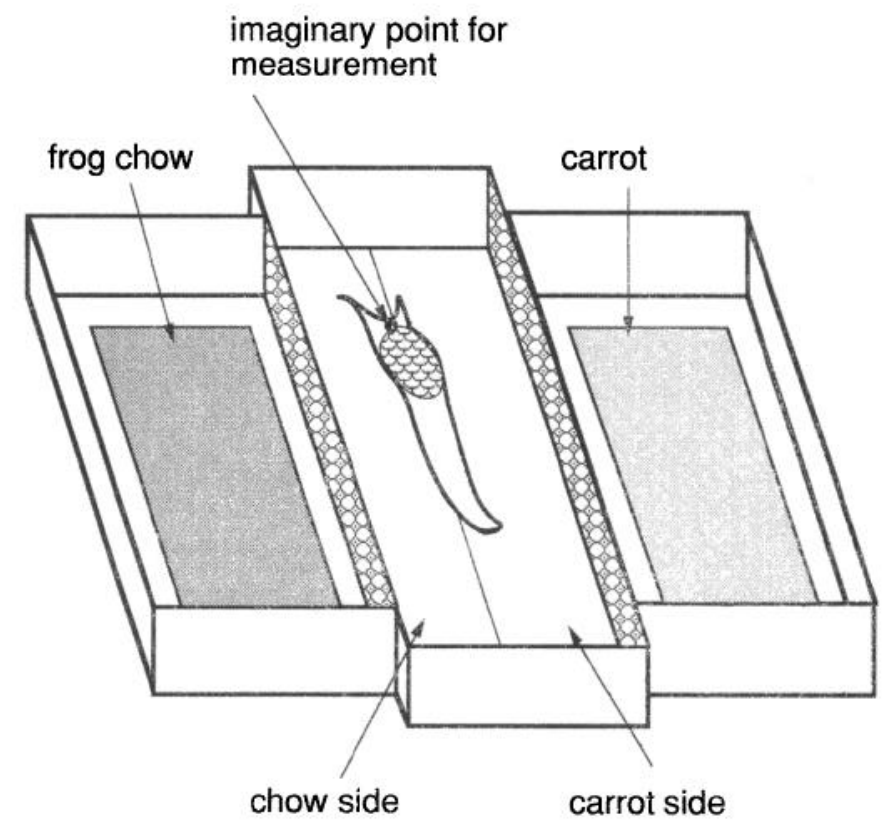

Figure 1. Testing chamber for the measurement of "carrot preference time." The odor sources of carrot and frog chow were placed in opposite side chambers. The slug was placed in the center chamber, the walls of which were perforated. A center line divided the center room into a "carrot side" and a "chow side." The carrot preference time was the percentage of time each slug's head spent on the carrot side during three testing trials (120 sec observation/testing trial). The dot on the head of the slug was the point used for measurement.

in Fig. 1 as a small dot, was used as a measurement marker). We did not control the initial head position, but it was distributed equally on both sides. Most slugs ( $\sim 85 \%)$ showed choice behavior, where they waved their head or tentacles before selecting one side. However, some slugs crawled through the center chamber and some stopped moving. For the measurement, we did not take into account whether slugs showed choice behavior or not. Although this contributed to a larger deviation in the time spent on the carrot side, our aim was to exclude any judgement bias made by the experimenters. If the slug's head did not cross the center line during the first $30 \mathrm{sec}$, the testing trial was restarted even when the slug seemed to show an odor-based movement decision. However, this rarely occurred ( $<5 \%)$ and crossing was observed within two additional testing trials.

Each slug underwent three testing trials at $2 \mathrm{hr}$ intervals (= one test session). The experimenter did not know the experimental treatments experienced by the slug being tested. The tests were carried out on the day following the training, unless specifically mentioned.

The parameter used for assessment of conditioning, "carrot preference time," was the percentage of time each slug's head spent on the carrot side. This was obtained by dividing the total time each slug's head spent on the carrot side by the total measured time in one test session (120 $\sec \times 3$ measurements $=360 \mathrm{sec}$ ).

Statistical analysis. Analysis of variance (ANOVA) was used to compare the odor choices of the groups. Newman-Keuls post hoc tests were used for further analysis of the data when $F$ ratios reached significance. We also used $t$ test for analyzing differences in the odor choices between two groups.

\section{Results}

\section{Cooling-induced retrograde amnesia in Limax flavus}

First, we examined whether retrograde amnesia was induced by cooling in Limax flavus conditioned to avoid food odor. Fiftynine slugs were divided into six groups. Slugs in groups (CQ) $1 \mathrm{~F}$ $(n=9),(\mathrm{CQ}) 2 \mathrm{~F}(n=11),(\mathrm{CQ}) 3 \mathrm{~F}(n=9)$, and (CQ)4F $(n=9)$ received $1,2,3$, and $4 \mathrm{CQ}$ training pairs, respectively, and were cooled immediately after the last training trial. Slugs in group 


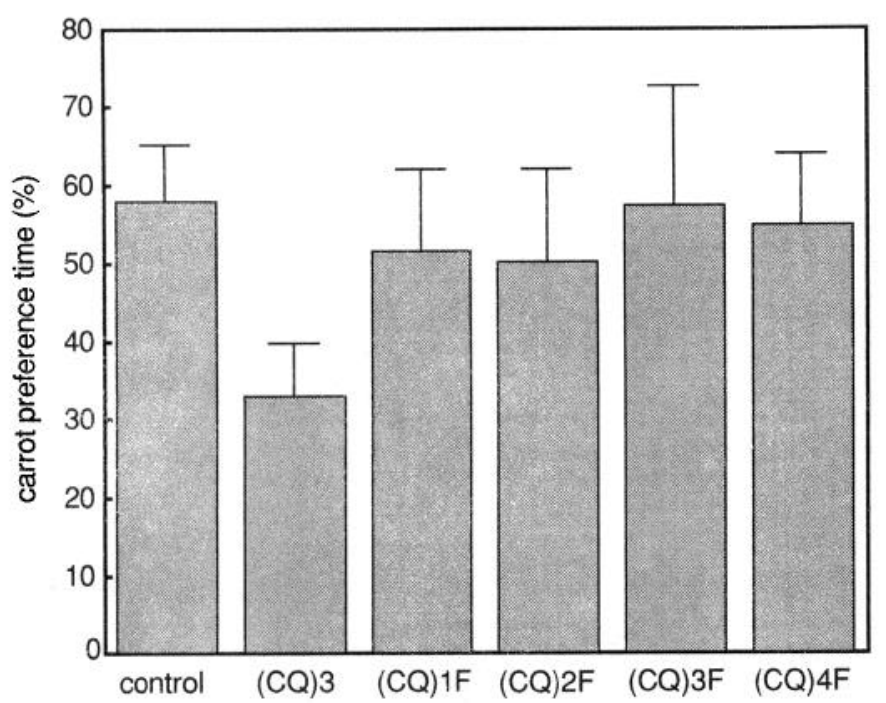

Figure 2. Retrograde amnesia induced by cooling in Limax flavus. Slugs in groups (CQ) $1 \mathrm{~F}(n=9),(\mathrm{CQ}) 2 \mathrm{~F}(n=11),(\mathrm{CQ}) 3 \mathrm{~F}(n=9)$, and $(C Q) 4 \mathrm{~F}(n=9)$ received $1,2,3$, and 4 training CQ pairs, respectively, and were cooled immediately after the trial. Slugs in group (CQ) $3(n=$ 12) received three training CQ pairs and were not cooled. Slugs in the control group $(n=9)$ were exposed to carrot juice and quinidine sulfate solution three times each; however, the ISI was $15 \mathrm{~min}$. Error bars represent SD.

(CQ)3 $(n=12)$ and the control group $(n=9)$ underwent the procedures described in Materials and Methods. As shown in Figure 2, the slugs in groups $(\mathrm{CQ}) 1 \mathrm{~F},(\mathrm{CQ}) 2 \mathrm{~F},(\mathrm{CQ}) 3 \mathrm{~F}$, and (CQ)4F showed similar carrot preference times to those in the control group. ANOVA revealed that there were differences among the groups $[F(5,53)=5.009 ; p<0.025]$. Individual comparison indicated that group (CQ) 3 was significantly different from the others $(p<0.05)$ and that there were no differences among groups $(\mathrm{CQ}) 1 \mathrm{~F},(\mathrm{CQ}) 2 \mathrm{~F},(\mathrm{CQ}) 3 \mathrm{~F},(\mathrm{CQ}) 4 \mathrm{~F}$, and the control $(p>0.25)$.

As reported by Sahley et al. (1981a), a slug was able to learn to avoid carrot odor with only one CQ pair. This observation indicates that a slug could easily learn an interevent relationship and also suggests that the cooling might have some logical significance for the slug and work to break the CS-US relationship. To examine this possibility, 18 slugs were divided into three groups, control $(n=6),(\mathrm{CQ}) 3(n=6)$, and (CF) $3(n=6)$. Slugs in group (CQ) 3 and the control group were treated in the same ways as those in Figure 2. For the slugs in group (CF)3, cooling was used as US instead of quinidine sulfate. With carrot juice as $\mathrm{CS}$, three conditioning trials were applied (ITI $=2 \mathrm{hr}$ ), and the results are shown in Figure 3. The carrot preference time of slugs in group (CF)3 was not reduced. ANOVA revealed that there were differences among the groups $[F(2,15)=3.975 ; P<$ 0.05 ]. Individual comparison indicated that group (CF) 3 was significantly different from group (CQ)3 $(P<0.05)$, and no difference was revealed between group (CF)3 and the control group $(p>0.1)$. Thus, slugs in group (CF) 3 did not learn to avoid carrot odor. These results suggest that the cooling did not have any logical significance for the slug and that the cooling itself did not break the CS-US relationship.

From these observations, we concluded that the recovery in carrot preference time shown in Figure 2 was a result of retrograde amnesia. In the rest of the study, the amnesia was used as a tool for distinguishing memory states in Limax flavus.

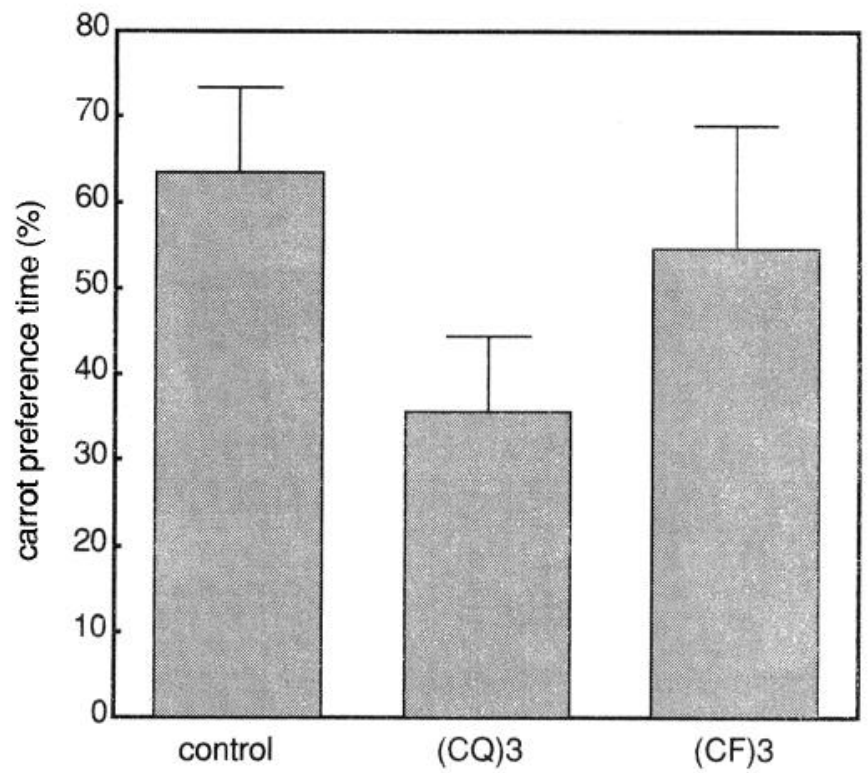

Figure 3. The effect of cooling as an unconditioned stimulus. Slugs in the control group $(n=6)$ and $(\mathrm{CQ}) 3,(n=6)$ received the same treatments as those in Figure 2. Slugs in group (CF) $3(n=6)$ received three training trials with carrot juice as $\mathrm{CS}$ and cooling $\left(\sim 1^{\circ} \mathrm{C}, 5 \mathrm{~min}\right)$ as US. Error bars represent SD.

\section{Early process of memory formation}

In the experiments described below, the temporal evolution of the slug's memory state was studied using the cooling-induced retrograde amnesia. The first experiment was designed to reveal the early process of memory formation. Forty-four slugs were divided into seven groups. Slugs in group $\mathrm{T}$ received a single CQ training trial and were cooled for $5 \min$ from $T$ min [ $T=$ $0(n=6), 0.5(n=7), 1(n=6), 2(n=6)$ and $5(n=6)]$ after the end of the trial. Slugs in group CQ $(n=7)$ also received a single training trial but were not cooled. Slugs in the control group $(n=6)$ received a 2 min exposure to carrot juice and then a $1 \mathrm{~min}$ exposure to saturated quinidine sulfate solution with $30 \mathrm{~min}$ ISI. The results are shown in Figure 4. As the interval between $\mathrm{CQ}$ and $\mathrm{F}$ became longer, a shorter carrot preference time was observed. ANOVA indicated that there were differences among the groups $[F(6,37)=3.87 ; p<0.01]$. Individual comparison (Newman-Keuls test) revealed that group CQ was significantly different from the control and groups $T=0,0.5$, and $1(p<0.05)$.

These results show that there are at least two memory states in the early process of memory formation, one sensitive and the other insensitive to cooling. It was also apparent that transition from the cooling-sensitive to the cooling-insensitive state occurred within $1 \mathrm{~min}$ after the training trial. We will call the former memory state "STM" and the latter "LTM."

\section{Reactivated state of LTM}

In the next experiments, we studied changes in the memory state in the LTM. Fifty-eight slugs were divided into seven groups. Slugs in group T were conditioned with three CQ training pairs and then cooled for $5 \mathrm{~min}$ from $T \min [T=0(n=8)$, $0.5(n=7), 1(n=7), 2(n=8)$, and $5(n=10)]$ after the end of the last training trial. Slugs in group (CQ)3 $(n=8)$ also received three $C Q$ pairs but were not cooled. Slugs in the control 


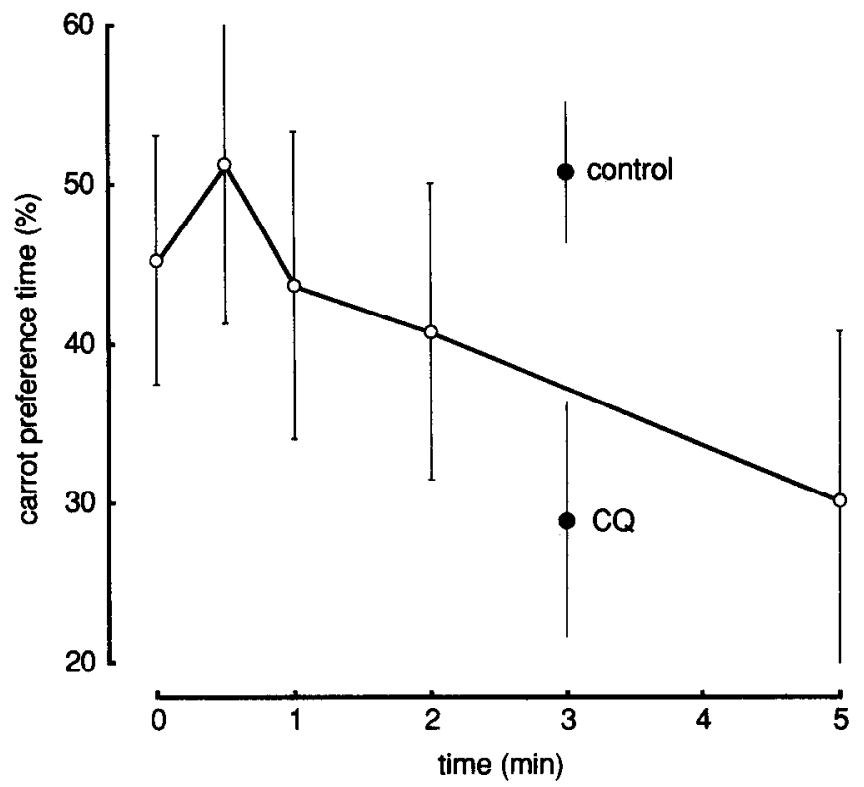

Figure 4. Carrot preference time as a function of CQ-F interval. Slugs in groups $T=0(n=6), 0.5(n=7), 1(n=6), 2(n=6)$, and $5(n=$ 6) received a single $C Q$ training pair and were cooled for $5 \mathrm{~min}$ from $0,0.5,1,2$, and 5 min after the end of quinidine treatment, respectively (open circles). Solid circles, carrot preference time of slugs in group $C Q$ $(n=7)$, which also received a single training trial, and that of slugs in the control group $(n=6)$, which rcceived a single unpaired CQ treatment (ISI $=30 \mathrm{~min})$. Error bars represent SD.

group ( $n=10$ ) were exposed to carrot juice three times and to quinidine sulfate three times as described in Materials and Methods. The ITI of the conditioning was $2 \mathrm{hr}$, and the memory was of the long-tcrm form within $1 \mathrm{~min}$ after conditioning. Thus, it was confirmed that the memory was of the long-term form when the conditioned slugs were cooled. The results are shown in Figure 5. As the interval between the last $C Q$ and $F$ was increased, a shorter carrot preference time was obtained. ANOVA indicated differences among the groups $[F(6,51)=3.255 ; p$ $<0.01]$. Individual comparison revealed that group $(\mathrm{CQ}) 3$ was significantly different from the control and groups $T=0,0.5$, and $1(p<0.05)$, and that the control group was significantly different from groups $T=2$ and $5(P<0.05)$. Thus, it was suggested that there are at least two memory states in LTM, one sensitive and the other insensitive to cooling.

In food-aversive conditioning in Limax maximus, the slug was able to learn to avoid food odor even with a single training trial (Sahley et al., 1981a). This was also the case for Limax flavus, as shown in group CQ of Figure 3. In addition, slugs conditioned with a single CQ training trial showed a similar carrot preference time to those conditioned with three CQ training trials (compare the carrot preference time of group $C Q$ in Fig. 4 with that of group (CQ)3 in Fig. 5). These results suggest that the second or third CQ pair applied to the slugs in group $T$ of Figure 5 worked not only to reinforce the conditioning but also to reactivate the memory trace. This means that a set of treatments that reactivate the memory trace followed by cooling, that is, CQF in Figure 5, must be necessary to induce amnesia. The following experiment was carried out to examine this possibility.

Fifty-seven slugs were divided into three groups, control ( $n$ $=19), \mathrm{CQF}(n=19)$, and CQ-F $(n=19)$. Slugs in group CQF

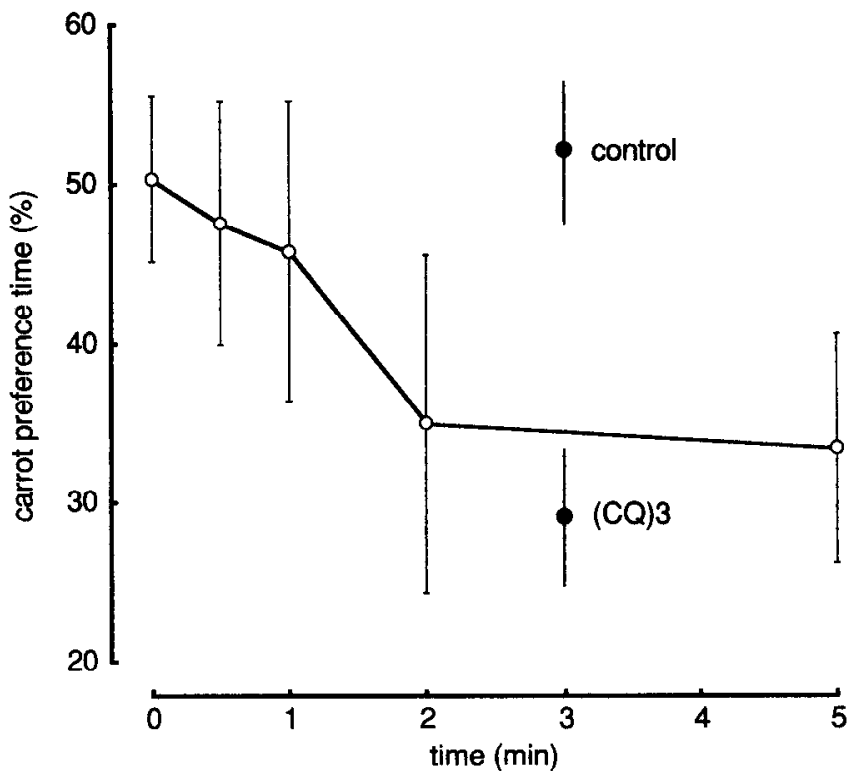

Figure 5. Carrot preference time as a function of (CQ)3-F interval. Slugs in groups $T=0(n=8), 0.5(n=7), 1(n=7), 2(n=8)$, and 5 $(n=10)$ were conditioned to avoid carrot odor with three CQ training pairs and were cooled for $5 \mathrm{~min}$ from $0,0.5,1,2$, and $5 \mathrm{~min}$ after the end of the third quinidine treatment, respectively (open circles). Solid circles, carrot preference time of slugs in groups $(C Q) 3(n=8)$ and the control group $(n=10)$, which received the same procedure as in Figure 2. Error bars represent SD.

and CQ-F were conditioned with two CQ training pairs and then tested for their carrot odor preference on the day of training. Six hours later, the slugs in group CQF were cooled immediately after an additional CQ training pair. Slugs in group CQ-F were cooled for $5 \mathrm{~min}$ from $15 \mathrm{~min}$ after the end of the additional CQ pair. These slugs were tested for their carrot odor preference again on the following day. Slugs in the control group received the same treatments as those in Figure 3, and their carrot preference was tested on the day after the treatment. The results are shown in Table 1. Comparison of individual carrot preference times showed that, with CQF, the time increased in 17 of 19 animals, whereas it increased in 10 of 19 given the CQ-F treatment. Statistical analysis $(t$ test) indicated that the slugs in group CQF recovered their carrot odor preference as a result of CQF treatment $[t(18)=3.959 ; p<0.001]$, and no difference was

Table 1. Comparison of individual carrot preference time before and after CQF treatment

Carrot preference time (\%)

\begin{tabular}{llllll}
\cline { 3 - 5 } Group & $n$ & $\begin{array}{l}\text { Before } \\
\text { CQF/CQ-F }\end{array}$ & $\begin{array}{l}\text { After } \\
\text { CQF/CQ-F }\end{array}$ & $\begin{array}{l}\text { In- } \\
\text { creased }^{b}\end{array}$ & $\begin{array}{l}\text { De- } \\
\text { creased }^{b}\end{array}$ \\
\hline Control & 19 & $54.7 \pm 8.7$ & & & \\
CQF & 19 & $30.9 \pm 8.4$ & $50.1 \pm 13.7$ & 17 & 2 \\
CQ-F & 19 & $31.7 \pm 7.1$ & $35.9 \pm 12.0$ & 10 & 9 \\
\hline
\end{tabular}

Slugs in groups CQF and CQ-F were conditioned with two CQ training pairs and their carrot preference time was measured on the day of the training. After they had been treated with CQF (CQ-F interval $=0 \mathrm{~min}$ ) or CQ-F (CQ-F interval $=15$ $\mathrm{min}$ ), their carrot preference time was measured again. Slugs in the control group received the same treatments as in Figure 2.

${ }^{a}$ Average $\pm \mathrm{SD}$.

${ }^{b}$ Number of slugs. 


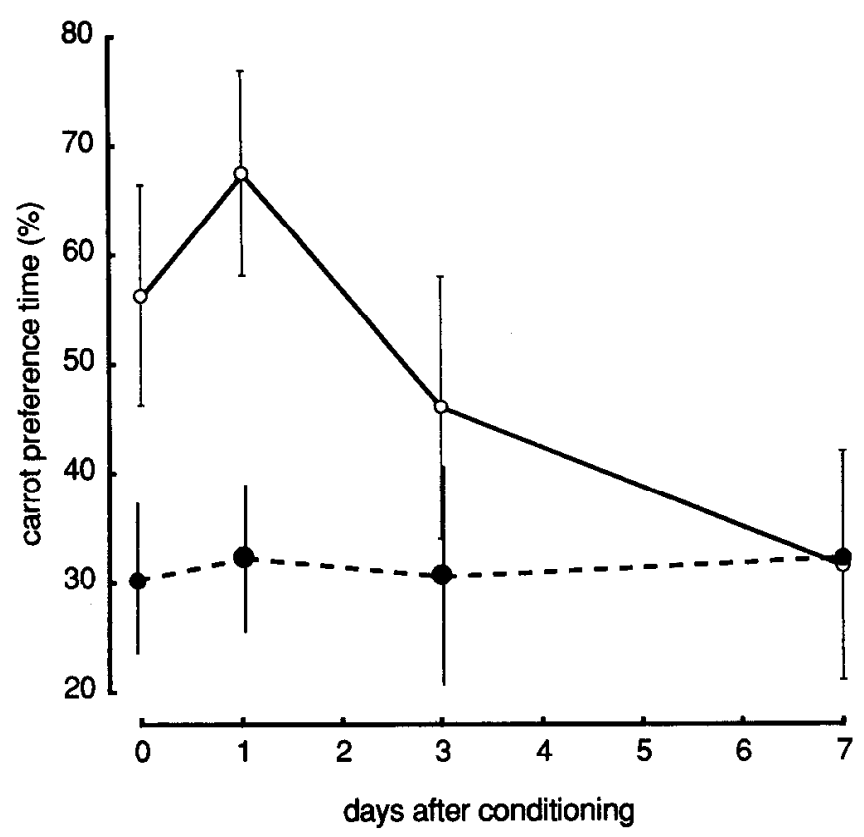

Figure 6. Carrot preference time as a function of (CQ)3-CQF interval. Slugs in groups $T=0(n=6), 1(n=6), 3(n=6)$, and $7(n=6)$ were conditioned to avoid carrot odor on day 0 with three $C Q$ conditioning pairs and were treated with $\mathrm{CQF}$ on days $0,1,3$, and 7 , respectively (open circles). Another set of slugs (four groups; $n=6$ each) also received three CQ training pairs, and their carrot odor preference was measured on days $0,1,3$ and 7 (solid circles). Error bars represent SD.

observed in the carrot preference time before and after the CQ-F treatment $[t(18)=1.012 ; p>0.2]$. The carrot preference time of group CQF after the CQF treatment was not different from that of the control group $(P>0.2)$.

If CQF treatment is effective because CQ reactivates the LTM and the cooling is introduced while the memory trace is reactivated, other stimuli capable of reactivating the memory trace should be also effective. In the case of FOC, CS is a good candidate. Therefore, we examined whether carrot juice plus cooling treatment (CF) induced amnesia in conditioned slugs. Thirty slugs were divided into three groups. Slugs in groups (CQ) $2 \mathrm{CF}$ $(n=9)$ and (CQ)3CF $(n=10)$ were conditioned to avoid carrot odor with two and three $C Q$ training trials, respectively, and then tested for their carrot odor preference. Next, they were exposed to carrot juice, followed immediately by $5 \mathrm{~min}$ cooling and then tested again. Slugs in the control group $(n=11) \mathrm{re}-$ ceived the same treatments as those in Table 1 . The results are shown in Table 2. Comparison of individual carrot preference times showed that, with CF treatment, the time was increased in 7 of 9 animals in group (CQ) $2 \mathrm{CF}$ and in 9 of 10 animals in group (CQ)3CF. Statistical analysis indicated that slugs recovered their carrot odor preference as a result of the CF treatment in group $(\mathrm{CQ}) 2 \mathrm{CF}[t(8)=2.825 ; p<0.05]$ and group $(\mathrm{CQ}) 3 \mathrm{CF}$ $[t(9)=2.619 ; p<0.05]$. The carrot preference time after the $\mathrm{CF}$ treatment in group (CQ) $2 \mathrm{CF}$ and group (CQ) $3 \mathrm{CF}$ showed no difference from that of the control group $(p>0.25)$.

These results clearly indicate that reactivation of the memory trace was necessary for induction of amnesia in Limax flavus and that the time-dependent curve in Figure 5 represented the process of reactivation of the memory trace. Thus, the two LTM
Table 2. Comparison of individual carrot preference time before and after CF treatment

\begin{tabular}{lrllll} 
& \multicolumn{5}{l}{ Carrot preference time (\%) } \\
\cline { 3 - 6 } Group & \multicolumn{1}{c}{$n$} & Before CF $^{a}$ & After CF $^{a}$ & $\begin{array}{l}\text { In- } \\
\text { creased }^{b}\end{array}$ & $\begin{array}{l}\text { De- } \\
\text { creased }^{b}\end{array}$ \\
\hline Control & 11 & $52.7 \pm 9.1$ & & & \\
(CQ)2CF & 9 & $30.8 \pm 8.4$ & $52.9 \pm 18.8$ & 7 & 2 \\
(CQ)3CF & 10 & $30.6 \pm 8.6$ & $48.0 \pm 15.3$ & 9 & 1
\end{tabular}

Slugs in groups (CQ) $2 \mathrm{CF}$ and (CQ) $3 \mathrm{CF}$ received two and three CQ training pairs, respectively, and their carrot time preference time was measured on the day of the training. After they had been treated with CF (C-F interval $=0 \mathrm{~min}$ ), their carrot preference time was measured again. Slugs in the control group received the same treatments as in Figure 2.

a Average \pm SD.

${ }^{b}$ Number of slugs.

states described above corresponded to the reactivated state and so-called the resting state.

\section{Long-term stabilization of LTM}

In the last experiment, we studied the changes in memory state using a longer time scale. Slugs were conditioned to avoid carrot odor and then were cooled on particular days after the training. Twenty-four slugs were conditioned to avoid carrot odor with three CQ training pairs. Then they received CQF treatment on the day of the training $(n=6)$ or $1 \mathrm{~d}(n=6), 3 \mathrm{~d}(n=6)$, or $7 \mathrm{~d}$ after $(n=6)$. The slugs were tested for their carrot odor preference on the day of CQF treatment. Another set of 24 slugs were divided into four groups (six slugs/group) and conditioned in the same way. With these groups we observed the retention of memory as a control. Thus, their carrot odor preferences were measured on the same day as the CQF treatment in the CQFtreated groups. As shown in Figure 6, amnesia was effectively induced by CQF treatment on the day of training $[t(10)=4.73$; $p<0.001]$ or on the day after $[t(10)=6.19 ; p<0.001]$. However, the treatment became less effective on the third day $[t(10)$ $=2.19 ; 0.05<p<0.1]$. On the seventh day, there was no difference between the carrot preference time of the CQF-treated group and that of the untreated control $[t(10)=0.324 ; p>0.5]$.

These results indicate that, within a few days after training, the memory changes its state so as to become insensitive to cooling-induccd amnesia.

\section{Discussion}

We found that retrograde amnesia could be induced by cooling of the terrestrial mollusk, Limax flavus, which was conditioned to avoid a food odor. Using the amnesia, temporal evolution of memory states were observed. In the early process of memory acquisition, two states were distinguishable, STM and LTM. The lifetime of the STM was about $1 \mathrm{~min}$. The LTM was reactivated with external stimuli such as the CS or CS-US training pair. The lifetime of the reactivated state of LTM was also about $1 \mathrm{~min}$. Within a few days after training, the LTM became resistant to cooling-induced amnesia.

\section{Cooling as a tool for amnesia induction}

There have been many studies on experimentally induced retrograde amnesia in a variety of animals. As well as surgical treatment or drugs, ECS has been most frequently employed to induce the amnesia (Miller and Springer, 1973). Carbon dioxide or nitrogen gas has also been used for this purpose, but Limax 
shows an aversive response to these agents (Gelperin, 1975). Therefore, we used cooling in order to distinguish amnesia from some logical operations that might break the CS-US relation acquired by the slug. In fact, Limax has been reported to show such logical operations, an extinction or US preexposure effect, where only CS or US applied to the conditioned slugs breaks the CS-US relation (Sahley et al., 1981a; Gelperin et al., 1985). If cooling has some logical significance for the slug, treatment with CQF or CF will decrease the CS-US contingency and the slugs will not show conditioned responses. However, this was not the case in the present study, because groups with the same CS-US contingency showed different results. For example, slugs in group $T=5$ in Figure 5 showed similar carrot odor preference to slugs in group $T=5$ in Figure 4 . However, their CS-US contingencies were different (three CS-US pairing plus one cooling vs. one CS-US pairing plus one cooling). In addition, the cooling itself was a neutral stimulus for the slug (Fig. 3). Thus, we conclude that there is no correlation between the observed recovery in carrot odor preference and the amount of CS-US contingency and that the observed recovery is a result of retrograde amnesia.

\section{Temporal evolution of memory states}

From the results shown in Figures 4-6, we offer an explanation for the memory states and their transitions in Limax flavus. In the early process of acquisition, memory exists in a form where cooling can induce amnesia (STM). After $1 \mathrm{~min}$ or so, the memory changes its state to one that is resistant to cooling (LTM). External stimuli can reactivate the LTM to the form that is sensitive to cooling. The lifetime of the reactivated state of the LTM is also about 1 min. Within a few days after the conditioning, the LTM changes its state again to one that is resistant to cooling, even if it is reactivated, and this is retained for about 1 month (T. Sekiguchi, A. Yamada, H. Suzuki, A. Mizukami, unpublished observation).

This scheme agrees well with the experimental results reported by many researchers. For the early process of memory acquisition, it is widely accepted from many experimental data that there are two distinct memory states (e.g., Duncan, 1949; Hudspeth and McGaugh, 1964; Weissman, 1964; Taber and Banuazizi, 1966; Leukel and Quinton, 1964; Riddle, 1969; Erber, 1976). In the present study, two memory states, STM and LTM, were also identified in the early process of memory acquisition in the food-aversive conditioning of Limax flavus, as shown in Figure 4. There have been many reports on the lifetime of STM, but they have differed each other depending on animals, conditioning procedures, or agents that induced amnesia, for example, $10 \mathrm{sec}$ (rat, avoidance conditioning and $\mathrm{CO}_{2}$ narcosis; Leukel and Quinton, 1964), $3 \mathrm{~min}$ (honeybee, color-discriminative learning and cooling; Erbcr, 1976), or $40 \mathrm{~min}$ (rat, leverpressing in Skinner box and ECS; Weissman, 1964). The lifetime of STM obtained in this study, about $1 \mathrm{~min}$, was relatively but not unusually short. Although longer lifetime for STM (1 d) was reported from behavioral or electrophysiological studies in $A p l y$ sia (Pinsker et al., 1973), they cannot be directly compared with those because of differences in experimental strategies.

The reactivated state of LTM (Fig. 5; Tables 1, 2) has also been reported in a variety of animals, for example, the rat (Misanin et al., 1968; Neilson, 1968), goldfish (Davis and Klinger, 1969), and fly (Quinn and Dudai, 1976). For example, Misanin et al. (1968) carried out experiments similar to ours and showed that retrograde amnesia was induced by ECS in a rat if it was applied immediately after several training trials or after the presentation of CS used in the training. They pointed out the importance of memory reactivation for the induction of amnesia. Our scheme can reflect a similar mechanism in the evolution of memory states.

According to Squire and Spanis (1984), ECS was effective for inducing amnesia within 2 weeks after learning in the mouse. In other words, ECS was able to induce loss of memory acquired within the previous 2 weeks. A similar property has also been reported in humans (Barbizet, 1970). These results correspond to ours (Fig. 6). As described abovc, this is interpreted as the evolution to a memory state that is resistant to amnesia even if reactivated.

Some previous experimental results seem to contradict the above scheme. In the case of color-discriminative learning in a honeybee (Erber, 1975), the insect shows an interesting relationship in learning index, (CS-US) $k=(\mathrm{CS}-\mathrm{US}) k+1 \cdot \mathrm{ECS}$. That is, bees conditioned with $k+1$ pairs of CS-US followed immediately by ECS show a similar learning index to those conditioned with $k$ pairs of CS-US. This means that ECS selectively induces amnesia only on newly acquired memory and suggests that the reactivated state of LTM is resistant to amnesia. However, the phenomenon can also be explained if the transition from LTM to stabilized LTM is very fast in the case of the honeybee.

It is possible that the durations of various phases are dependent on the efficacy of training. In fact, it has been reported that three learning mutants of Drosophila show different time courses in STM-LTM memory transition as compared to normal Drosophila (Dudai et al., 1976; Duerr and Quinn, 1982; Dudai, 1983; Quinn and Greenspan, 1984). These mutants (dunce, rutabaga, and amnesic) show appreciable initial learning, but all have unusually rapid memory decay for the first hour afterward. Similar results were obtained in a selected line of poor-learning honeybee, Apis mellifera capensis (Brandes et al., 1988). Because we had no learning-deficient mutants or lines of Limax flavus, we were unable to study how the durations of various phases depend on the efficiency of learning. Therefore, we intend to carry out further experiments to study this dependence.

The present study provides a total picture of the evolution of memory states during associative learning in the terrestrial slug Limax flavus, and the results agreed well with those observed in a variety of animals. The similarity in the changes of memory state suggests that the basic mechanisms of learning and memory are very similar. The origin of the memory states and the mechanism of their evolution will be studied electrophysiologically or biochemically in the future.

\section{References}

Baily CH, Chen M (1988) Long-term memory in Aplysia modulates the total number of varicosities of single identified sensory neurons. Proc Natl Acad Sci USA 85:2373-2377.

Barbizet J (1970) Human memory and its pathology. San Francisco: Freeman.

Brandes CH, Frisch B, Menzel R (1988) Time-course of memory formation in honey bee lines selected for good and poor learning. Anim Behav 36:981-985.

Byrne JH (1987) Cellular analysis of associative learning. Physiol Rev $67: 329-439$.

Carew TJ, Sahley CL (1986) Invertebrate learning and memory: from behavior to molecules. Annu Rev Neurosci 9:435-487.

Crow T (1983) Conditioned modification of locomotion in Hermissenda crassicornis: analysis of time-dependent associative and nonassociative components. J Neurosci 3:2621-2628. 
Crow T, Alkon DL (1978) Retention of an associative behavioral change in Hermissenda. Science 219:397-400.

Davis RE, Klinger PD (1969) Environmental control of amnesic effects of various agents in goldfish. Physiol Behav 4:269-271.

Dudai Y (1983) Mutations affect storage and use of memory differentially in Drosophila. Proc Natl Acad Sci USA 80:5445-5448.

Dudai Y, Jan Y, Byers D, Quinn WG, Benzer S (1976) dunce, a mutant of Drosophila deficient in learning. Proc Natl Acad Sci USA 73:1684 1688

Duerr JS, Quinn WG (1982) Three Drosophila mutations that block associative learning also affect habituation and sensitization. Proc Natl Acad Sci USA 79:3646-3650.

Duncan CP (1949) The rctroactive effect of electroshock on learning. J Comp Physiol Psychol 42:32-44.

Erber J (1975) The dynamics of learning in the honeybee (Apis mellifera carnica). II. Principles of information processing. J Comp Physiol 99:243-255.

Erber J (1976) Retrograde amnesia in honeybees (Apis mellifera carnica). J Comp Physiol Psychol 90:41-46.

Freckleton WC Jr, Wahlstan D (1968) Carbon dioxide-induced amnesia in the cockroach. Psychon Sci 12:179-180.

Frost WN, Castellucci VF, Hawkins RD, Kandel ER (1985) The monosynaptic connections from the sensory neurons participate in the storage of long-term memory for sensitization of the gill- and siphon-withdrawal refiex in Aplysia. Proc Natl Acad Sci USA 82: 8266-8269.

Gelperin A (1975) Rapid food-aversion learning by a terrestrial mollusk. Science 189:567-570.

Gelperin A, Tank DW, Hopfield JJ (1985) The logic of Limax learning. In: Model neural networks and behavior (Selverson AI, ed), pp 237262. New York: Plenum.

Heriot JT, Coleman PD (1962) The effect of electroconvulsive shock on retention of a modified "one-trial" conditioned avoidance. J Comp Physiol Psychol 55:1082-1084.

Hudspeth WJ, McGaugh JL (1964) Aversive and amnesic effects of electroconvulsive shock. J Comp Physiol Psychol 57:61-64.

Leukel F, Quinton E (1964) Carbon dioxide effects on acquisition and extinction of avoidance behavior. J Comp Physiol Psychol 57:267270.

McGaugh JL (1966) Time-dependent process in memory storage. Science 153:1351-1358.

Menzel R (1984) Short-term memory in bees. In: Primary neural substrates of learning and behavior change (Alkon DL, Farley J, eds), pp 259-274. Cambridge, MA: Cambridge UP.
Miller RR, Springer AD (1973) Amnesia, consolidation, and retrieval. Psychol Rev 80:69-79.

Misanin JR, Miller RR, Lewis DJ (1968) Retrograde amnesia produced by electroconvulsive shock after reactivation of a consolidated memory trace. Science 160:554-555.

Montarolo PG, Goelet P, Catellucci VF, Morgan J, Kandel ER, Schacher S (1986) A critical time window for macromolucular synthesis in long-term heterosynaptic facilitation in Aplysia. Science 234:12491254.

Neilson HC (1968) Evidence that electroconvulsive shock alters memory retrieval rather than memory consolidation. Exp Neurol 20:320.

Pinsker HM, Henning WA, Carew TJ, Kandel ER (1973) Long-term sensitization of a defensive withdrawal reflex in Aplysia. Science 182: 1039-1042.

Quinn WG, Dudai Y (1976) Memory phases in Drosophila. Nature 262:576-577.

Quinn WG, Greenspan RJ (1984) Learning and courtship in Drosophila: two stories with mutants. Annu Rev Neurosci 7:67-93.

Riddle WI (1969) Effect of electroconvulsive shock: permanent or temporary retrograde amnesia. J Comp Physiol Psychol 67:140-144.

Sahley CL, Gelperin A, Rudy JW (1981a) One-trial associative learning modifies food odor preferences of a terrestrial mollusc. Proc Natl Acad Sci USA 78:640-642.

Sahley CL, Rudy JW, Gelperin A (1981b) An analysis of associative learning in a terrestrial mollusc. I. Higher-order conditioning, blocking and a transient US pre-exposure effect. J Comp Physiol 144:1-8.

Sahley CL, Rudy JW, Gelperin A (1984) Associative learning in a mollusk: a comparative analysis. In: Primary neural substrates of learning and behavioral change (Alkon DL, Farley J, eds), pp 243258. Cambridge, MA: Cambridge UP.

Sahley CL, Martin KA, Gelperin A (1990) Analysis of associative learning in the terrestrial mollusc Limax maximus. II. Appetitive learning. J Comp Physiol A 167:339-345.

Squire LR (1987) Memory and brain. New York: Oxford UP.

Squire LR, Spanis CW (1984) Long gradient of retrograde amnesia in mice: continuity with the findings in humans. Behav Neurosci 98: 345-348.

Taber RI, Banuazizi A (1966) $\mathrm{CO}_{2}$-induced retrograde amnesia in a one-trial learning situation. Psychopharmacologia (Berlin) 9:382-391.

Weissman A (1964) Retrograde amnesia effect of supramaximal electroconvulsive shock on one-trial acquisition in rats: a replication. $J$ Comp Physiol Psychol 57:248-250. 